\title{
BMJ Open Quality Reducing redundant creatine kinase testing in cardiac injury
}

\author{
Sheharyar Raza (D) , ${ }^{1}$ Andre C Amaral, ${ }^{2}$ Jeffrey Pang, ${ }^{3}$ Fuad Moussa, ${ }^{4}$ \\ Dominick Shelton, ${ }^{5}$ Lowyl Notario, ${ }^{5}$ Heather Harrington, ${ }^{6}$ Jeannie L Callum,,${ }^{7,8}$ \\ Paul M Yip (D) 7,8
}

To cite: Raza S, Amaral AC, Pang J, et al. Reducing redundant creatine kinase testing in cardiac injury. BMJ Open Quality 2020;9:e001182. doi:10.1136/ bmjoq-2020-001182

- Additional material is published online only. To view, please visit the journal online (http://dx.doi.org/10.1136/ bmjoq-2020-001182).

Received 25 August 2020 Revised 3 December 2020 Accepted 15 December 2020

Check for updates

(c) Author(s) (or their employer(s)) 2020. Re-use permitted under CC BY-NC. No commercial re-use. See rights and permissions. Published by BMJ.

For numbered affiliations see end of article.

Correspondence to

Dr Paul M Yip:

paul.yip@utoronto.ca

\section{ABSTRACT}

Background Creatine kinase (CK) testing in the setting of suspected cardiac injury is commonly performed yet rarely provides clinical value beyond troponin testing. We sought to evaluate and reduce CK testing coupled with troponin testing by $50 \%$ or greater.

Methods We performed root cause analysis to study prevailing processes and patterns of CK testing. We developed new institutional guidelines, removed CK from high-volume paper and electronic order bundles and conducted academic detailing for departments with highest ordering frequency. We evaluated consecutive patients at Sunnybrook Health Sciences Centre between 1 January 2018 and 31 March 2020 who had either a CK or troponin level measured. We prespecified successful implementation as a reduction of $50 \%$ in total CK orders and a decrease in the ratio of CK-to-troponin tests to onethird or less. We retained additional data beyond our study period to assess for sustained reductions in testing.

Results Total CK tests decreased over the study period from 3963 to 2111 per month, amounting to a $46.7 \%$ reduction $(95 \% \mathrm{Cl} 33.2$ to $60.2 ; \mathrm{p}<0.001)$ equalling 61 fewer tests per hospital day. Troponin testing did not significantly change during the intervention. Ratio of CKto-troponin tests decreased from 0.91 to $0.49(p<0.001)$. The reduction coincided with changes to order-sets, was observed across all clinical units and was sustained during additional months beyond the study period. These reductions in testing resulted in a projected annual cost savings of $\mathrm{C} \$ 28446$.

Conclusions We demonstrate that a low-cost and feasible quality improvement initiative may lead to significant reduction in unnecessary CK testing and substantial savings in healthcare costs for patients with suspected cardiac injury.

\section{INTRODUCTION}

Cardiac disease is a widespread cause of morbidity and mortality in Canada affecting 1 in 12 adults and accounting for about 1 in 5 of all deaths. ${ }^{1}$ Common mechanisms of injury include myocardial infarction, heart failure, pulmonary embolism, disorders of aorta and pericarditis. ${ }^{2}$ Rapid and reliable diagnosis of cardiac injury, particularly acute coronary syndromes, is essential for effective management. ${ }^{3}$ Serum biomarkers can provide valuable early clues to diagnosis and prognosis. Historical examples of biomarkers include aspartate transaminase, lactate dehydrogenase, creatine kinase (CK) and creatine kinase-MB isozyme (CK-MB). ${ }^{4}$ Since its introduction in the 2000 s, high-sensitivity cardiac troponin (henceforth troponin) has been shown to be the superior biomarker for rapid diagnosis of cardiac injury. Contrary to evidence, CK and CK-MB testing remains widespread even when troponin testing is available, leading to extraneous blood draws, diagnostic confusion and unnecessary healthcare expenditure. ${ }^{5}$

Troponin testing has been compared with CK-MB in several head-to-head trials examining diagnostic performance across a range of indications. Compared with CK-MB, troponin is more sensitive, specific and an earlier marker of myocardial injury. ${ }^{6-8}$ The diagnostic superiority of troponin extends to patients with renal failure, those with remote infarcts and those who experience cardiac reinfarction. ${ }^{9-11}$ Both CK-MB and troponin rise similarly in cardiac injury yet peak troponin levels provide equivalent estimates of infarct size evaluated post hoc using cardiac imaging. ${ }^{12} 13$ Uncertainty remains regarding the role of biomarker testing after cardiac procedures including percutaneous coronary intervention and coronary artery bypass grafts. ${ }^{14}$ Data are lacking for conclusive recommendations favouring either troponin or CK-MB, yet this subgroup represents a minority of indications for cardiac biomarker testing. ${ }^{15}$

Reflecting this body of evidence, Choosing Wisely (national body with a focus on healthcare resource stewardship) now solely recommends troponin as the biomarker of choice for myocardial injury where available. ${ }^{16}$ This recommendation is echoed in the American Heart Association and European Cardiology Association guidelines, and CK testing has been omitted from the global consensus definition of myocardial infarction. ${ }^{17-19}$ Notwithstanding contemporary guidelines, CK testing for cardiac injury remains widespread in Canadian hospitals. ${ }^{50-22}$ 
In this quality improvement initiative, we sought to evaluate and reduce CK testing for suspected cardiac injury by $50 \%$ or greater from existing levels. Our institution no longer performs CK-MB isozyme testing (discontinued in April 2014) while non-specific CK for cardiac testing has persisted despite the availability of troponin. ${ }^{23}$ Healthcare institutions elsewhere have adopted similar targets for CK-MB reduction initiatives and achieved lower false positive diagnoses, unchanged mortality from myocardial infarction, fewer blood draws and substantial cost-savings. ${ }^{24}$ Strategies have included cost-analysis of unnecessary testing, revision and dissemination of institutional guidelines, targeted educational presentations, changes to common investigation bundles and use of plan-do-study-act cycles. ${ }^{25}{ }^{26}$ We employed multimodal strategies with the goal of reducing unnecessary CK testing at a large Canadian academic cardiac centre.

\section{METHODS}

\section{Context}

We analysed all instances of troponin and CK orders for patients at Sunnybrook Health Sciences Centre because our institution is a large Canadian cardiac centre and offers a broad spectrum of services including emergency evaluation, percutaneous cardiac intervention, cardiovascular surgical services, acute trauma management and cardiovascular intensive care. Our study period spanned 1 January 2018 to 31 March 2020 yielding a comprehensive dataset reflecting 1 year before our intervention, 1 year after initiation and 3 additional months beyond. Enrolment was conducted under a waiver from ethics according to institutional guidelines as the study collected deidentified patient data, involved a quality improvement project and reported results as aggregate outcomes only.

\section{Root cause analysis}

We began by examining channels through which CK tests were ordered at our institution with the goal of targeting highest-volume channels first. Laboratory testing processes depended on clinical setting and included electronic entry (used primarily for emergency department and outpatient clinic settings), combined electronic and paper orders (used primarily for inpatient admissions and procedures) and paper orders (used primarily for inpatients on wards). These processes were standardised, applied consistently over the study duration and detailed in a previous investigation. ${ }^{27}$ Orders for cardiac biomarkers originated from diverse clinical settings, were entered into an electronic laboratory ordering system (OACIS, Telus Health, Montreal, Quebec, Canada), relayed to a laboratory information system (Sunquest, Tuscon, Arizona, USA) and final results made available to ordering physicians on electronic health record (SunnyCare) developed at our institution. Notably, recurring CK testing was part of standardised order bundles for cardiac admissions (such as for acute myocardial infarctions, ST-elevation myocardial infarctions and congestive heart failure), intensive care (such as for major trauma, rhabdomyolysis and sedation) and postcardiac procedure order sets (online supplemental appendix 4).

We also met individually with quality improvement leaders in high-volume CK testing specialties, including emergency medicine, cardiology and intensive care units to explore prevailing clinical reasoning for coupled CK and troponin testing in their contexts. Prior to the start of our intervention, suspicion of cardiac injury usually prompted biomarker testing for troponin and CK, which were frequently ordered together by the clinical team or via an electronic ordering bundle. For cardiac indications, CK was almost never ordered without troponin, however the converse (a troponin without a CK) did sometimes occur. Similarly, for non-cardiac conditions with suspected muscle damage (eg, rhabdomyolysis), a CK was usually ordered without a troponin, however the converse was rare. Finally, for conditions where both cardiac and non-cardiac muscle damage was suspected (eg, major trauma to thorax), both CK and troponin were almost always ordered together. A further use occurred in cases of subacute myocardial infarction, where a discordance between CK and troponin results (the former being normal and the latter abnormal) may provide clues about the timeline of cardiac injury. ${ }^{28}$ Finally, we identified several non-cardiac indications such as neurological, endocrine and musculoskeletal disorders, and our interventions did not address these conditions. $^{29}$

\section{Intervention}

Borrowing from past initiatives, our quality improvement approach had several pillars including detailed revision of institutional testing guidelines, pre-intervention analysis, targeted information sessions with key clinical and administrative stakeholders, modifications to testing processes, ongoing evaluation and feedback and postintervention audit for sustained changes. ${ }^{28}$ Our consulting team included representatives from the departments of laboratory medicine, haematology, general medicine, cardiology, cardiovascular surgery, emergency medicine, intensive care, nursing management and nursing education.

\section{Evaluation and revision of institutional guidelines}

We conducted a literature review of biomarker testing including primary research and clinical practice guidelines from prominent cardiology societies. Existing literature focused on CK-MB isozyme testing, as non-specific CK testing has inferior test characteristics for the diagnosis of cardiac injury. ${ }^{7}$ Recent studies also described prevailing myths about the value of CK testing and an informal survey of our colleagues indicated that these myths existed at our institution. ${ }^{28}$ Our revised institutional testing guidelines reflected clinical testing nuances and were widely publicised using our hospital intranet to disseminate evidence and solicit feedback. 


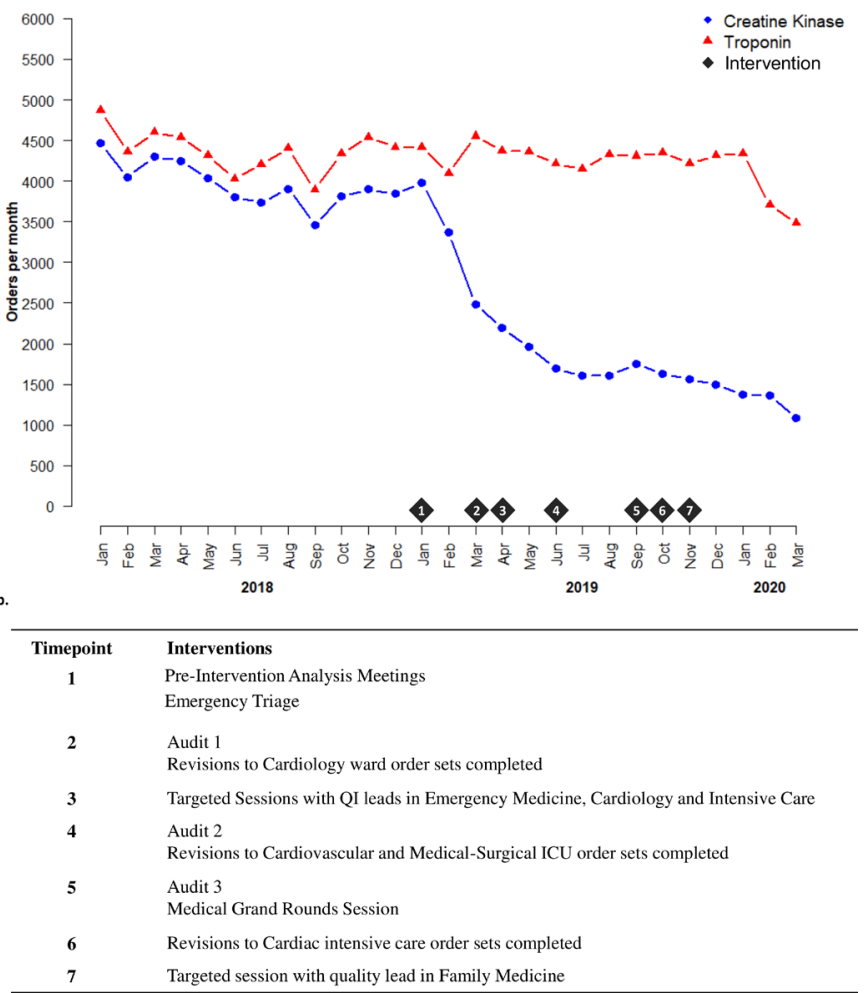

Figure 1 (A) Total creatine kinase and troponin orders per month during study period. Interventions were deployed in January 2019. (B) Intervention deployment by hospital ward and date. ICU, intensive care unit; QI, Quality Improvement.

\section{Pre-intervention analysis}

We evaluated the frequency of CK and troponin testing in the year preceding our intervention. Analysis focused on temporal patterns (weekday vs weekend), ordering physicians (by specialty), clinical unit (outpatient clinic, emergency department, cardiology ward, intensive care and other inpatient wards), coupled orders (simultaneous CK and troponin testing) and recurring orders (frequency and duration).

\section{Targeted lectures and information sessions}

Our team members conducted information, feedback and education sessions with aforementioned stakeholders throughout the intervention period. Large group engagement involved audiences from nursing education, laboratory medicine, family medicine and city-wide grand rounds presentation to share our message broadly (figure 1). ${ }^{28}$

\section{Modifications to testing processes}

Beginning in January 2019, we implemented several changes to the testing process grounded in behavioural principles of quality improvement. We began by removing CK testing from emergency department triage order bundles, standard cardiology admission order sets, standard cardiovascular surgery and intensive care orders (online supplemental appendix 5). We reasoned that changing defaults may lead to behaviour change without placing restrictions on testing. ${ }^{30}$ After repeat audit of testing in May 2019, we intervened on specific units where CK testing frequency remained high. For these units, we provided individualised comparisons with similar clinical settings as a nudge to change testing practices. In specific instances, such as testing for propofol infusion syndrome, we reduced default intervals of recurring CK tests reasoning that changing the reference point for testing frequency (e.g. from every 8 hour to once daily) might lead to more conscious decisions about repeated blood draws. ${ }^{31}$ Importantly, none of our interventions prohibited clinicians from ordering a CK when they made a deliberate decision to test.

\section{Ongoing evaluation and feedback}

Our team conducted ongoing quarterly audit and review to assess our process measures of CK testing: total monthly CK tests, CK-to-troponin ratio and testing frequency by clinical unit. We did not declare a balancing measure. Guided by audit data, we contacted nurse managers and departmental quality leaders to provide individualised email feedback on monthly testing frequency and CK-totroponin ratio until a sustained reduction was achieved.

\section{Measures}

We measured the total number of CK and troponin tests monthly from 1 January 2018 to 31 December 2019 (n=24 months) to reflect 1 year prior and 1 year following our intervention. Three additional months of data from 1 January 2020 to 31 March 2020 were collected to check for sustained reduction. To account for lag in full implementation, we separately analysed data after all order sets were updated (1 October 2019). We calculated the ratio of CK-to-troponin tests ordered reasoning that the number of troponin tests reflected instances where cardiac injury was suspected. We also calculated the number of coupled CK and troponin orders, because this number more closely reflected CK testing for cardiac injury on a caseby-case basis. Additionally, we computed occurrences of consecutive tests defined as sequences of three or more tests ordered with $<25$ hours between each test, because CK is often ordered as a recurring test (online supplemental appendix 1). To assess economic impact, we derived cost per test from an estimate of reagent, analyzer and personnel costs estimated in previous research at our institution (C\$1.28 per test). ${ }^{27}$ 32-34

\section{Statistical analysis}

Our statistical plan involved analysis of CK and troponin tests comparing individual frequency and ratio in the year preceding and following intervention. Changes in these measures were calculated using conditional logistic regression. We made further comparisons of CK and troponin by ordering physicians, hospital location, coupled tests and consecutive tests. Wilcoxon rank-sum test or $\chi^{2}$ test was applied for comparing testing before and after intervention. Two-tailed $p$ value $<0.05$ was considered statistically significant. We used run charts to 
assess and graphically present change in testing over the study period. ${ }^{35}$ All analyses were conducted using Statistical Analysis Software (V.9.4 for Windows, Cary, North Carolina, USA) and R package (V.3.6.1).

\section{RESULTS}

In the year preceding our intervention (1 January 2018 to 31 December 2018), a total of 47556 CK tests were performed (monthly median of 3905; IQR 3809-4147) and 52506 troponin tests were performed (monthly median of 4381; IQR 4259-4541). The CK-to-troponin ratio was 0.91 and $72.8 \%$ of total CK tests were coupled with troponin (table 1). Consecutive CK tests accounted for $36.4 \%$ of all tests. The top $20 \mathrm{CK}$ ordering physicians accounted for $26.0 \%$ of all tests ordered, and mainly comprised emergency physicians, cardiologists and intensivists (online supplemental appendix 3). The top CK ordering units accounted for a high proportion of all

Table 1 Summary statistics for creatine kinase (CK) and troponin testing before and after intervention

\begin{tabular}{|c|c|c|}
\hline & $\begin{array}{l}\text { Before intervention } \\
\text { (January-December 2018) }\end{array}$ & $\begin{array}{l}\text { After intervention } \\
\text { (January-December 2019) }\end{array}$ \\
\hline \multicolumn{3}{|l|}{ CK orders } \\
\hline \multicolumn{3}{|l|}{ Monthly orders } \\
\hline Mean \pm SD & $3963 \pm 276$ & $2111 \pm 795^{\star}$ \\
\hline Total orders $(n=115722)$ & 52506 & 51691 \\
\hline \multicolumn{3}{|l|}{ Monthly orders } \\
\hline Mean \pm SD & $4376 \pm 259$ & $4308 \pm 124$ \\
\hline Median (Q1, Q3) & $4381(4259,4541)$ & $4319(4217,4368)$ \\
\hline \multicolumn{3}{|l|}{ Coupled tests $\ddagger$} \\
\hline Total (\% of total CK orders) & $34625(72.81 \%)$ & $14769(58.30 \%)^{*}$ \\
\hline \multicolumn{3}{|l|}{ Consecutive CK tests } \\
\hline \multicolumn{3}{|l|}{ Monthly consecutive CK tests } \\
\hline Mean $\pm S D$ & $1444 \pm 144$ & $758 \pm 341^{\star}$ \\
\hline \multicolumn{3}{|l|}{ Consecutive troponin tests $\S$} \\
\hline \multicolumn{3}{|c|}{ Monthly consecutive troponin tests } \\
\hline Mean $\pm S D$ & $1950 \pm 158$ & $1989 \pm 61$ \\
\hline Emergency & $25875(49.28 \%)$ & $24939(48.25 \%)^{\star}$ \\
\hline Cardiologyף & $5169(9.84 \%)$ & $5278(10.21 \%)$ \\
\hline Intensive care ${ }^{\star \star}$ & $11268(21.46 \%)$ & $11447(22.15 \%)$ \\
\hline All other units & $10194(19.41 \%)$ & $10027(19.40 \%)$ \\
\hline
\end{tabular}

Data are count (percentage) of each column unless noted as median (IQR).

${ }^{*}$ Significant at $\mathrm{p}<0.001$.

†CK divided by troponin.

¥Simultaneously ordered CK and troponin tests.

$\S$ Two or more tests on the same patient $<24$ hours apart.

ISummed counts from cardiology ward, cardiac intensive care and post-procedure unit.

**Summed counts from cardiovascular, general and neurological intensive care units. 


\section{Before Intervention}
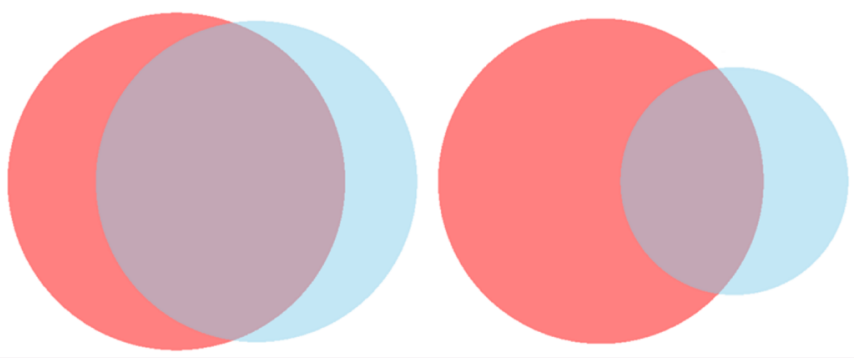

Troponin

Creatine kinase

Coupled

\begin{tabular}{lcc}
\hline & $\begin{array}{c}\text { Before Intervention } \\
\text { (Jan-Dec 2018) }\end{array}$ & $\begin{array}{c}\text { After Intervention } \\
\text { (Jan-Dec 2019) }\end{array}$ \\
\hline Creatine Kinase Orders & & \\
$\quad$ Total & 47,556 & 25,332 \\
$\quad$ Monthly (blue circle) & 3,963 & 2,111 \\
\hline Troponin Orders & & \\
$\quad$ Total & 52,506 & 51,691 \\
$\quad$ Monthly (red circle) & 4,376 & 4,308 \\
\hline Coupled Orders & & \\
$\quad$ Total & 34,625 & 14,769 \\
\hline Monthly (purple overlap) & 2,885 & 1,231 \\
\hline
\end{tabular}

Figure 2 Total orders for creatine kinase (blue), troponin (red) and coupled tests (purple overlap) before and after intervention.

tests performed and included emergency department (43.8\%), intensive care $(26.7 \%)$ and cardiology units $(8.7 \%)$.

During the year of our intervention (1 January 2019 to 31 December 2019), a total of 25332 CK tests were performed (monthly median of 1721; IQR 1608-2340) equaling a reduction of $46.7 \%$ (95\% CI $33.2 \%$ to $60.2 \%$ ), or about 61 fewer tests per hospital day (figure 1). During the same period, a total of 51691 troponin tests were performed (monthly median 4319; IQR 4217-4368) equaling an overall decrease of $1.6 \%$ which was not statistically significant $(\mathrm{p}=0.356)$. This resulted in a CK-to-troponin ratio of 0.49 . Coupled orders decreased to $58.3 \%$ of total CK tests equaling $57.3 \%$ reduction $(\mathrm{p}<0.001)$ (figure 2 ). Subgroup analyses revealed substantial reductions in CK testing across all departments; in particular, our emergency department $(70 \%)$, intensive care unit $(40 \%)$ and cardiology units (10\%) all saw substantial reductions (all $\mathrm{p}$ values $<0.001$ ). Top 20 ordering physicians accounted for $33.1 \%$ of all tests in the postintervention period with no substantial change in specialty composition. These improvements remained in separate analyses after full implementation of order sets changes and were sustained in the three additional months following our study period (1 January 2020 to 31 March 2020) (online supplemental appendix 2).

Analysis of financial impact showed an estimated cost of C\$5072.64 per month in the year before intervention and $\mathrm{C} \$ 2702.08$ per month in the year after, amounting to a potential savings of $\mathrm{C} \$ 28446.72$ annually.

\section{DISCUSSION}

We evaluated CK testing in the setting of cardiac injury and implemented quality improvement interventions to reduce unnecessary testing. Our initiative led to a statistically significant reduction in total and coupled CK tests, and a decrease in CK-to-troponin ratio from 0.91 to 0.49 . Reductions were observed across all clinical units and sustained over additional months, reflecting a stable change in CK ordering at our institution. Our interventions did not target troponin testing and, as expected, it did not significantly change during our study period. A diverse change team and targeted outreach to departments with high-volume testing allowed us to achieve substantial reductions in areas with the greatest potential impact. The use of behavioural defaults, informational nudges and reference point changes were key influences in changing ordering behaviour without explicit prohibitions that may restrict clinical flexibility. To our knowledge, our study is the first of its kind in Canada targeting cardiac biomarker testing, the first to evaluate the CK in this context and could potentially layout a roadmap for similar improvements at other institutions.

Several major cardiac care centres elsewhere have reported similar quality improvement initiatives to eliminate routine CK-MB testing in cardiac injury. Larochelle et al. developed revised institutional guidelines and removed CK-MB from computerised order sets. They observed a $66 \%$ reduction in CK-MB testing, US $\$ 1.25$ million in cost savings and no change in incidence of acute coronary syndrome (ACS) at 1year follow-up. ${ }^{24}$ Ducatman et al. used time-sequenced interventions to reduce CK-MB testing which lead to improved guideline compliance from $32 \%$ to $58 \% .^{20}$ They additionally found that CK-MB testing failed to detect additional myocardial infarctions and lead to 42 preventable admissions later found not to be myocardial infarctions. Other authors have implemented similar protocols to reduce CK-MB testing to achieve sustained reductions of $80 \%-95 \%$ and considerable cost savings. ${ }^{36} 37$ Our study adapted several practices from these protocols for our institution.

Our interventions to reduce testing had several unique contextual features. We used strategies tailored for multiple laboratory processes at our institution, which involved two electronic and one paper-based avenue for orders. The diversity of channels made ongoing audits more demanding, for example, paper order sets were printed in batch and full implementation required removing old versions from circulation while electronic orders required special requests for modification. These factors may make our findings more generalisable to institutions both with and without electronic ordering systems. ${ }^{38}$ Our institution had precedent for quality improvement in laboratory testing and quality leaders from diverse specialties with an openness to change initiatives. ${ }^{27} 3940$ The study setting was a large academic centre with frequent educational events which allowed for creative opportunities to disseminate information, yet created challenges in knowledge translation due to high 
turnover of medical trainees. Implementation in nonacademic settings may require other tailored efforts to gain traction.

The study has several limitations. Our institution uses CK testing rather than cardio-specific isozyme CK-MB as used in other studies. Studies of clinical outcomes resulting from test reductions have focused on CK-MB and are not easily extrapolated to our study, however we believe that CK has lower accuracy and would be even less likely to adversely impact care. ${ }^{8}$ Institutions that may have eliminated the CK-MB might still carry CK testing for other indications and our work highlights the need to evaluate the value of CK testing for cardiac injury in the era of much improved troponin assays. Our overall test reduction of $46.7 \%$, although within margin of error, fell short of our a priori goal of $50 \%$ reduction in overall CK testing. This may be partly attributable to non-cardiac indications for CK testing which were not affected by our intervention. We also experienced delays in revising all cardiology order bundles due to an initial lack of consensus on testing guidelines, and later clerical errors in rollout of updated order bundles. This resulted in a delay before all cardiology units carried new order sets, which may underestimate testing reductions and limited follow-up duration to 8 months on these units (online supplemental appendices 2-4). Additionally, the volume of testing for suspected cardiac injury may have changed over the study period. However, our study was not designed for such adjustments to outcome measures.

Our study does not incorporate other measures of cardiac injury such as ECG, echocardiography, MRI and cardiac catherisation, which are additional sources of valuable clinical information. Appropriateness of such tests can be the focus of future projects. The intervention lead to fewer laboratory tests, costs and unnecessary blood draws, however we did not evaluate impact on clinical events such as iatrogenic anaemia, hospital stay or death, and cannot provide specific such insights about patient outcomes. Our study follow-up period was limited, and the data were collected concurrently with our intervention. This could lead to underestimation of our effect in the short term and overestimation in the long term, although a comparable past intervention at our institution has led to sustained changes. ${ }^{27}$

Our study has several strengths which could be emulated in future projects. We used a novel metric, the CK-totroponin ratio, which normalises CK testing frequency over troponin orders and may be adopted as a quality metric for institutions to track CK testing across time and differing clinical contexts. We included consecutive patients with no exclusions. This allowed for a comprehensive sample size and rich dataset for geographic and temporal analysis of testing patterns. Our study is a pragmatic implementation of quality improvement which lead to immediate benefits at our institution. Although our design cannot establish causality, it provides stronger evidence of real-world feasibility than controlled experiments. The interventions preserved clinician autonomy in ordering laboratory tests they deemed necessary. We believe this aspect of our design was crucial for acceptance by clinical teams and successful execution of our project.

\section{CONCLUSION}

Creatine kinase testing in cardiac injury has little supportive evidence yet remains entrenched. A multimodal approach using educational, behavioural and analytic interventions can achieve swift and sustained reductions in unnecessary testing. This approach can be feasibly applied to achieve substantial cost-savings across healthcare institutions.

\section{Author affiliations}

${ }^{1}$ Internal Medicine, University of Toronto, Toronto, Ontario, Canada

${ }^{2}$ Interdepartmental Division of Critical Care, University of Toronto, Sunnybrook Health Sciences Centre, Toronto, Ontario, Canada

${ }^{3}$ Cardiology, Sunnybrook Health Sciences Centre, Toronto, Ontario, Canada

${ }^{4}$ Cardiac and Vascular Surgery, Sunnybrook Health Sciences Centre, Toronto, Ontario, Canada

${ }^{5}$ Emergency Medicine, Sunnybrook Health Sciences Centre, Toronto, Ontario, Canada

${ }^{6}$ Nursing Education, Sunnybrook Health Sciences Centre, Toronto, Ontario, Canada ${ }^{7}$ Laboratory Medicine and Molecular Diagnostics, Sunnybrook Health Sciences Centre, Toronto, Ontario, Canada

${ }^{8}$ Laboratory Medicine and Pathobiology, University of Toronto, Toronto, Ontario, Canada

\section{Twitter Paul M Yip @paulyip18}

Acknowledgements The authors would like to thank Liying Zhang for performing statistical analysis and creating figures.

Contributors SR, JC, PY, JP, FM, LN, AA, HH and DS helped with planning and execution of interventions. SR, JC and PY wrote the manuscript with input from all authors.

Funding The authors have not declared a specific grant for this research from any funding agency in the public, commercial or not-for-profit sectors.

Competing interests None declared.

Patient consent for publication Not required.

Provenance and peer review Not commissioned; externally peer reviewed.

Data availability statement Data are available on reasonable request. Data are available on individual request from the corresponding author PY.

Author note A low-cost quality improvement intervention may lead to substantial reductions in unnecessary CK testing for cardiac injury by @JeannieCallum and colleagues.

Supplemental material This content has been supplied by the author(s). It has not been vetted by BMJ Publishing Group Limited (BMJ) and may not have been peer-reviewed. Any opinions or recommendations discussed are solely those of the author(s) and are not endorsed by BMJ. BMJ disclaims all liability and responsibility arising from any reliance placed on the content. Where the content includes any translated material, BMJ does not warrant the accuracy and reliability of the translations (including but not limited to local regulations, clinical guidelines, terminology, drug names and drug dosages), and is not responsible for any error and/or omissions arising from translation and adaptation or otherwise.

Open access This is an open access article distributed in accordance with the Creative Commons Attribution Non Commercial (CC BY-NC 4.0) license, which permits others to distribute, remix, adapt, build upon this work non-commercially, and license their derivative works on different terms, provided the original work is properly cited, appropriate credit is given, any changes made indicated, and the use is non-commercial. See: http://creativecommons.org/licenses/by-nc/4.0/.

ORCID iDs

Sheharyar Raza http://orcid.org/0000-0002-8247-1484 
Paul M Yip http://orcid.org/0000-0002-7635-7407

\section{REFERENCES}

1 Statistics Canada. CANSIM table 102-0529, deaths, by cause, chapter IX: diseases of the circulatory system (100 to 199), age group and sex. Available: https://www150.statcan.gc.ca/t1/tbl1/en/ tv.action?pid=1310014701

2 Wu C, Singh A, Collins B, et al. Causes of troponin elevation and associated mortality in young patients. Am J Med 2018;131:284-92.

3 Bugiardini R, Ricci B, Cenko E, et al. Delayed care and mortality among women and men with myocardial infarction. J Am Heart Assoc 2017;6:e005968.

4 Lee TH, Goldman L. Serum enzyme assays in the diagnosis of acute myocardial infarction. recommendations based on a quantitative analysis. Ann Intern Med 1986;105:221-33.

5 Wiens EJ, Arbour J, Thompson K, et al. Routine creatine kinase testing does not provide clinical utility in the emergency department for diagnosis of acute coronary syndromes. BMC Emerg Med 2019;19:37.

6 Kavsak PA, MacRae AR, Newman AM, et al. Effects of contemporary troponin assay sensitivity on the utility of the early markers myoglobin and CKMB isoforms in evaluating patients with possible acute myocardial infarction. Clin Chim Acta 2007;380:213-6.

7 Macrae AR, Kavsak PA, Lustig V, et al. Assessing the requirement for the 6-hour interval between specimens in the American heart association classification of myocardial infarction in epidemiology and clinical research studies. Clin Chem 2006;52:812-8.

8 Adams JE, Abendschein DR, Jaffe AS. Biochemical markers of myocardial injury. Is MB creatine kinase the choice for the 1990s? Circulation 1993:88:750-63.

9 le EHY, Klootwijk PJ, Weimar W, et al. Significance of acute versus chronic troponin T elevation in dialysis patients. Nephron Clin Pract 2004;98:c87-92

10 Smulders MW, Bekkers SCAM, Kim HW, et al. Performance of CMR methods for differentiating acute from chronic MI. JACC CardiovasC Imaging 2015;8:669-79.

11 Thygesen K, Alpert JS, White HD, et al. Universal definition of myocardial infarction. Eur Heart J 2007;28:2525-38.

12 Ingkanisorn WP, Rhoads KL, Aletras $\mathrm{AH}$, et al. Gadolinium delayed enhancement cardiovascular magnetic resonance correlates with clinical measures of myocardial infarction. J Am Coll Cardiol 2004;43:2253-9.

13 Younger JF, Plein S, Barth J, et al. Troponin-I concentration $72 \mathrm{H}$ after myocardial infarction correlates with infarct size and presence of microvascular obstruction. Heart 2007;93:1547-51.

14 Jaffe AS, Apple FS, Lindahl B, et al. Why all the struggle about CK$\mathrm{MB}$ and $\mathrm{PCl}$ ? Eur Heart $J$ 2012;33:1046-8.

15 Lim CCS, van Gaal WJ, Testa L, et al. With the "universal definition," measurement of creatine kinase-myocardial band rather than troponin allows more accurate diagnosis of periprocedural necrosis and infarction after coronary intervention. J Am Coll Cardiol 2011;57:653-61.

16 American Society for Clinical Pathology. Myoglobin or ck-mb testing? Choosing Wisely [Internet], 2015. Available: http://www. choosingwisely.org/clinician-lists/american-society-clinicalpathology-myoglobin-to-diagnose-acute-myocardial-infarction/

17 Amsterdam EA, Wenger NK, Brindis RG, et al. 2014 AHAVACC Guideline for the Management of Patients With Non-ST-Elevation Acute Coronary Syndromes. J Am Coll Cardiol 2014;64:e139-228.

18 Collinson P, Hammerer-Lercher A, Suvisaari J, et al. How well do laboratories adhere to recommended clinical guidelines for the management of myocardial infarction: the cardiac marker guidelines uptake in Europe study (CARMAGUE). Clin Chem 2016;62:1264-71.

19 Thygesen K, Alpert JS, Jaffe AS, et al. Fourth universal definition of myocardial infarction (2018). J Am Coll Cardiol 2018;72:2231-64.
20 Ducatman AM, Tacker DH, Ducatman BS, et al. Quality improvement intervention for reduction of redundant testing. Acad Pathol 2017;4:237428951770750.

21 Le RD, Kosowsky JM, Landman AB, et al. Clinical and financial impact of removing creatine kinase-MB from the routine testing menu in the emergency setting. Am J Emerg Med 2015;33:72-5.

22 Choosing Wisely at Trillium Health Partners [Internet], 2019. Available: https://choosingwiselycanada.org/perspective/diving-intrillium-health-partners/

23 Mula CT, Human N, Middleton L. An exploration of workarounds and their perceived impact on antibiotic stewardship in the adult medical wards of a referral hospital in Malawi: a qualitative study. BMC Health Serv Res 2019;19:64.

24 Larochelle MR, Knight AM, Pantle H, et al. Reducing excess cardiac biomarker testing at an academic medical center. $J$ Gen Intern Med 2014;29:1468-74.

25 Solomon DH, Hashimoto H, Daltroy L, et al. Techniques to improve physicians' use of diagnostic tests: a new conceptual framework. JAMA 1998;280:2020-7.

26 Baird G. The laboratory test utilization management toolbox. Biochem Med 2014;24:223-34

27 Strauss R, Cressman A, Cheung M, et al. Major reductions in unnecessary aspartate aminotransferase and blood urea nitrogen tests with a quality improvement initiative. BMJ Qual Saf 2019;28:809-16.

28 Alvin MD, Jaffe AS, Ziegelstein RC, et al. Eliminating creatine Kinase-Myocardial band testing in suspected acute coronary syndrome: a value-based quality improvement. JAMA Intern Med 2017;177:1508-12.

29 Leverenz D, Zaha O, Crofford LJ, et al. Causes of creatine kinase levels greater than $1000 \mathrm{IU} / \mathrm{L}$ in patients referred to rheumatology. Clin Rheumatol 2016;35:1541-7.

30 Perry C, Chhantralia K, Damesick D. Behavioural insights in health care. The Health Foundation. Available: https://www.health.org.uk/ publications/behavioural-insights-in-health-care

31 Patel MS, Volpp KG. Leveraging insights from behavioral economics to increase the value of health-care service provision. J Gen Intern Med 2012;27:1544-7.

32 Henderson J, Bouck Z, Holleman R, et al. Comparison of payment changes and choosing wisely recommendations for use of low-value laboratory tests in the United States and Canada. JAMA Intern Med 2020;180:524-31.

33 Ministry of Health and Long Term Care. Schedule of benefits: physician services under the health insurance act. Amended October 1, 2005. Available: http://www.health.gov.on.ca/en/pro/programs/ ohip/sob/lab/lab_mn2020.pdf [Accessed 10 Aug 2020].

34 Gilmour JA, Weisman A, Orlov S, et al. Promoting resource stewardship: reducing inappropriate free thyroid hormone testing. $J$ Eval Clin Pract 2017;23:670-5.

35 Perla RJ, Provost LP, Murray SK. The run chart: a simple analytical tool for learning from variation in healthcare processes. BMJ Qual Saf 2011;20:46-51.

36 Baron JM, Lewandrowski KB, Kamis IK, et al. A novel strategy for evaluating the effects of an electronic test ordering alert message: optimizing cardiac marker use. J Pathol Inform 2012;3:3.

37 Sullivan P, Waymack J, Griffen D, et al. Effectively reducing CK-MB utilization using computer order entry in the emergency department. Am J Med Qual 2017;32:107.

38 Baron JM, Dighe AS. Computerized provider order entry in the clinical laboratory. J Pathol Inform 2011;2:35.

39 Etchells M, Spradbrow J, Cohen R, et al. Audit of appropriate use of platelet transfusions: validation of adjudication criteria. Vox Sang 2018;113:40-50.

40 Qiang JK, Thompson T, Callum J, et al. Variations in RBC and frozen plasma utilization rates across 62 Ontario community hospitals. Transfusion 2019;59:545-54. 